\title{
Geometric Phase Analysis of Strain in Naturally Deformed Olivine
}

\author{
C.L. Johnson, ${ }^{*}$ M.J. Hÿtch,** and P.R. Buseck*,***
}

* Dept. of Chemistry \& Biochemistry, Arizona State University, Tempe, AZ, 85287

** Centre d'Etudes de Chimie Métallurgique, CNRS, 15 rue G. Urbain, 94407 Vitry-sur-Seine, France

*** Dept. of Geological Sciences, Arizona State University, Tempe, AZ, 85287

Many tectonic processes are strongly influenced by the solid-state flow of minerals in Earth's upper mantle. One mechanism for this flow is plastic deformation, which typically involves the migration of dislocations through crystals. Dislocation structures can have profound effects on the mechanical and electrical properties of their host minerals. Recent innovations in image processing have provided a method for measuring the displacement field around dislocations at near-atomic scale [1]. We are developing this image-processing routine to measure the strain field around a dislocation in a naturally deformed olivine $\left[(\mathrm{Mg}, \mathrm{Fe})_{2} \mathrm{SiO}_{4}\right]$ from Earth's mantle.

A sample of mantle olivine from San Carlos, Arizona was investigated using a JEOL 4000EX. HREM images of dislocations in (101) tilt boundaries were collected. Most dislocations are weakly dissociated; however, some pure edge dislocations with $\mathbf{b}=[100]$ were observed. These dislocations were viewed end-on parallel to [010]. The images were digitized at high resolution and analyzed using routines written within DigitalMicrograph image-processing software.

The strain field is a function of the displacement of atoms around a dislocation core. Image formation in the core is complex, however just outside the core region, the image is, in many cases, easily interpretable and rows of spots can be directly related to crystallographic planes in the structure. In this region, the displacement of the image intensity from the regular array of spots can be determined to sub-Ångström resolution [1]. The displacement field is obtained by extracting the geometric phase information from the HREM image. The image contains both amplitude and phase components which store local contrast and positional information, respectively. The phase, once separated from the amplitude, is very sensitive to changes in the position of the image intensity. These variations can be related to the real-space displacements in the crystal and, in turn, the strain field around the dislocation. The details of the method are described fully elsewhere [2].

The HREM image in Figure 1a,b shows a [100] dislocation from a $\sim 1^{\circ}$ tilt boundary. The phase images (Fig. 2) were calculated from the (101) and (10-1) spots in the Fourier transform of the image (Fig. 1c). The dislocation is revealed by a $2 \pi$ discontinuity in the phase image that corresponds to a lattice displacement (extra half plane) in the crystal. The discontinuity terminates at the dislocation core. The results of the strain field analysis and comparison to the strain field generated by elastic theory will be presented [3].

[1] M.J. Hÿtch, in NATO ASI Series “Thermodynamics, Microstructure and Plasticity”, Fréjus 2002, Kluwer Academic Publishers, in press.

[2] M.J. Hÿtch, E. Snoeck and R. Kilaas, Ultramicroscopy 74 (1998) 131.

[3] This research was supported by NSF grant EAR-0003533. We thank H. Friedrich for helpful conversations. 

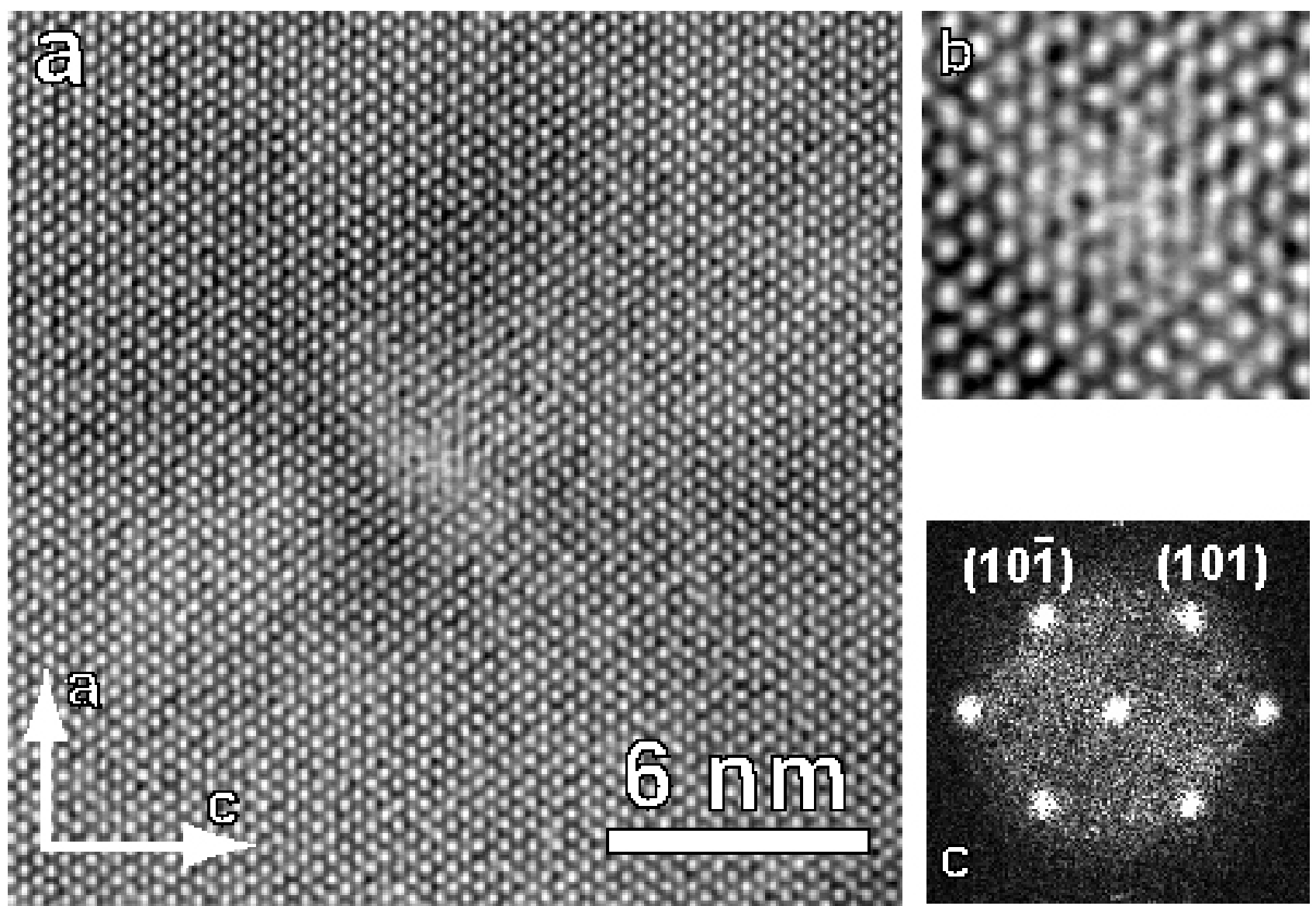

Figure 1. (a) HREM image of olivine in the [010] projection with $\mathbf{a} \mathbf{b}=[100]$ dislocation at the center. (b) Enlarged view of the dislocation core from 1a. (c) Fourier transform of 1a with (hkl) labels on the spots used for the geometric phase reconstruction.

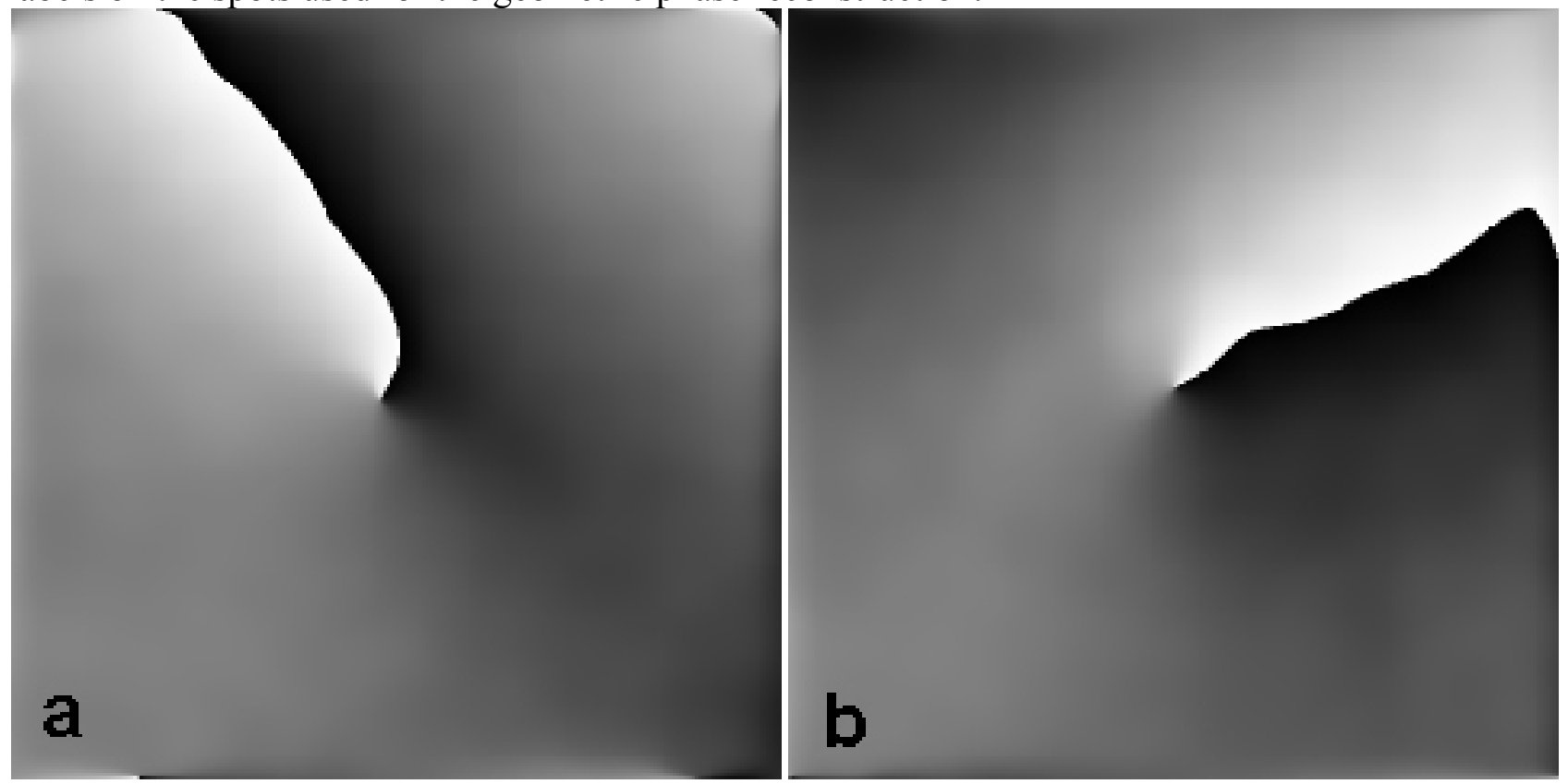

Figure 2. Geometric phase images from (a) the (101) and (b) the (10-1) spots in 1c. The sharp black/white boundaries are $2 \pi$ phase discontinuities resulting from the dislocation. 